\title{
PRIMARY DECOMPOSITIONS IN VARIETIES OF COMMUTATIVE DIASSOCIATIVE LOOPS
}

\author{
MICHAEL K. KINYON AND PETR VOJTĚCHOVSKÝ
}

\begin{abstract}
The decomposition theorem for torsion abelian groups holds analogously for torsion commutative diassociative loops. With this theorem in mind, we investigate commutative diassociative loops satisfying the additional condition (trivially satisfied in the abelian group case) that all $n$th powers are central, for a fixed $n$. For $n=2$, we get precisely commutative $C$ loops. For $n=3$, a prominent variety is that of commutative Moufang loops.

Many analogies between commutative $\mathrm{C}$ and Moufang loops have been noted in the literature, often obtained by interchanging the role of the primes 2 and 3 . We show that the correct encompassing variety for these two classes of loops is the variety of commutative RIF loops. In particular, when $Q$ is a commutative RIF loop: all squares in $Q$ are Moufang elements, all cubes are $C$ elements, Moufang elements of $Q$ form a normal subloop $M_{0}(Q)$ such that $Q / M_{0}(Q)$ is a C loop of exponent 2 (a Steiner loop), C elements of $L$ form a normal subloop $C_{0}(Q)$ such that $Q / C_{0}(Q)$ is a Moufang loop of exponent 3. Since squares (resp. cubes) are central in commutative $\mathrm{C}$ (resp. Moufang) loops, it follows that $Q$ modulo its center is of exponent 6 . Returning to the decomposition theorem, we find that every torsion, commutative RIF loop is a direct product of a C 2-loop, a Moufang 3-loop, and an abelian group with each element of order prime to 6 .

We also discuss the definition of Moufang elements, and the quasigroups associated with commutative RIF loops.
\end{abstract}

\section{INTRODUCTION}

A quasigroup $(Q, \cdot)$ is a set $Q$ with a binary operation $\cdot$ such that for each $a, b \in Q$, the equations $a x=b, y a=b$ have unique solutions $x, y \in Q$, respectively. A loop is a quasigroup with a neutral element 1, i.e., $1 x=x 1=x$ for every $x$. Basic references for quasigroups and loops are [2, 12.

A loop is power-associative if every element generates a subgroup (associative subloop), and diassociative if every two elements generate a subgroup. Powers $x^{n}$ are thus defined unambiguously in power-associative loops, and the order $|x|$ of $x$ can be introduced in the usual way.

For a power-associative loop $Q$ and a prime $p$, the p-primary component $Q_{(p)}$ is the set of all torsion elements $x \in Q$ such that $|x|$ is a power of $p$. A power-associative loop $Q$ is a $p$-loop if $Q=Q_{(p)}$.

A classical theorem of group theory states that every finitely generated torsion abelian group is a direct product of its $p$-primary components. For power-associative loops, a $p$-primary component need not even be a subloop. On the other hand, Bruck and Paige observed without proof in $[3]$ that the decomposition theorem holds in the variety of commutative diassociative loops. (We give a proof in 92 ,

In this paper, we investigate the situation when additional equational restrictions are imposed on the $p$-primary components of commutative diassociative loops.

The condition that all $n$th powers (for a fixed $n$ ) are central is trivially satisfied for commutative groups but not so for commutative diassociative loops, since the center of a loop consist of all elements that commute and associate with all other elements.

1991 Mathematics Subject Classification. Primary: 20N05.

Key words and phrases. commutative diassociative loop, commutative Moufang loop, commutative C loop, p-primary component, RIF loop, ARIF loop, Steiner loop, Steiner triple system, Moufang element, C element. 
In fact, the situation is fully understood only for the variety of commutative diassociative loops with squares in the center; this coincides with the variety of commutative $\mathrm{C}$ loops. A loop is called a $C$ loop if it satisfies the identity

$$
x(y \cdot y z)=(x y \cdot y) z .
$$

C loops satisfying $x \cdot y x=x y \cdot x$, which include the commutative ones, are diassociative [8].

The variety of commutative diassociative loops with cubes in the center includes commutative Moufang loops. A loop is called a Moufang loop if it satisfies any, and hence all, of the equivalent identities

$$
\begin{array}{cc}
x(y z \cdot x)=x y \cdot z x, & (x \cdot y z) x=x y \cdot z x, \\
x(y \cdot x z)=(x y \cdot x) z, & (z x \cdot y) x=z(x \cdot y x) .
\end{array}
$$

The diassociativity of Moufang loops is usually known as Moufang's Theorem [2, 12].

Already for $n=3$ do we find that the variety of commutative diassociative loops with central $n$th powers (for a fixed $n$ ) is rather unwieldy, because it properly contains the variety of commutative Moufang loops. For instance, from the general construction of Hart and Kunen [6], there exist nonMoufang, commutative diassociative loops of exponent 3 and order 27.

Thus, although the decomposition theorem for the variety of commutative diassociative loops with central $n$th powers is easy to prove (see 92 , it is not particularly useful, because this variety is too broad. Ideally, we would like to be able to characterize subvarieties of commutative diassociative loops whose $p$-primary components satisfy certain prescribed (equational) conditions. In general, however, this seems to be a difficult task.

In our previous work [13, we observed many analogies between commutative $\mathrm{C}$ loops and commutative Moufang loops, with $p=2$ playing a prominent role in the $\mathrm{C}$ case and $p=3$ in the Moufang case. For instance, as we have already noted, squares of elements in a commutative $\mathrm{C}$ loop are central, while cubes of elements in a commutative Moufang loop are central. In addition, a commutative $\mathrm{C}$ loop is a direct product of an abelian group and a commutative $\mathrm{C}$ 2-loop, while a commutative Moufang loop is a direct product of an abelian group and a commutative Moufang 3-loop. The present work was in part motivated by our desire to better understand this analogy.

It turns out that the behavior of commutative $\mathrm{C}$ and commutative Moufang loops can be described uniformly in the variety of commutative diassociative loops whose 2-primary component is $\mathrm{C}$ and whose 3-primary component is Moufang. More importantly, the encompassing variety happens to be the variety of commutative $R I F$ loops, i.e., inverse property loops satisfying either, and hence both, of the following identities:

$$
\text { (RIF1) } \quad(x y \cdot z) \cdot x y=x \cdot y(z x \cdot y),
$$

$$
x y \cdot(z \cdot x y)=(x \cdot y z) x \cdot y
$$

These loops were defined for the first time in 8 .

To understand the structure of commutative RIF loops requires the study of Moufang elements. These are traditionally defined (for well-motivated reasons) to be those elements $x$ satisfying either of the top two equations of (MFG) for every $y, z$. However, they could certainly be defined in other natural and non-equivalent ways, by fixing any variable in any one of the equations in (MFG), and assuming that the other two variables in that equation are universally quantified.

We analyze the situation in 93 , which we hope will eventually lead to a deeper understanding of Moufang elements. We could not resist the temptation and proved somewhat more than is needed for 4 , but the topic remains rife with open problems, some of which we state explicitly.

The main results of this paper can be found in 4 , where we describe the structure of commutative RIF loops and give the main decomposition theorem.

Finally, it is well-known that commutative Moufang loops are closely related to totally symmetric quasigroups, and commutative $\mathrm{C}$ loops to Steiner triple systems. As an application of our results, we conclude the paper in $\$ 5$ by showing how commutative RIF loops are related to a certain class of quasigroups, recovering the $\mathrm{C}$ and Moufang situations as special cases. 
Our investigations were aided by the automated theorem prover Prover9 [10, the finite model builder Mace4 [9], and the LOOPS package [11] for GAP [5].

\section{The General DECOMPOSITION}

A subloop $N$ of a loop $Q$ is normal, denoted $N \unlhd Q$, if it is a kernel of some loop homomorphism with domain $Q$. When $S$ is a subset of $Q$, we let $\langle S\rangle$ denote the subloop of $Q$ generated by $S$.

Let $\left\{Q_{i} \mid i \in I\right\}$ be a collection of subloops of a loop $Q$. Then $Q$ is the (internal) direct product of $\left\{Q_{i} \mid i \in I\right\}$ if

(i) $Q_{i} \unlhd Q$ for every $i$,

(ii) $Q_{i} \cap\left\langle Q_{j} \mid j \in I, j \neq i\right\rangle=1$,

(iii) $Q=\left\langle Q_{i} \mid i \in I\right\rangle$.

If the index set $I$ is finite, the internal direct product $Q$ of $\left\{Q_{i} \mid i \in I\right\}$ is isomorphic to the external direct product $\prod_{i \in I} Q_{i}$, where multiplication is performed componentwise ([2, Lemma IV 5.1]).

For a power-associative loop $Q$ and a positive integer $k$, let $Q_{[k]}$ denote the set of all torsion elements $x \in Q$ such that $|x|$ divides $k$.

Lemma 2.1. Let $Q$ be a commutative diassociative loop.

(i) For each $n \geq 0$, the mapping $Q \rightarrow Q ; x \mapsto x^{n}$ is a homomorphism with kernel $Q_{[n]}$.

(ii) For any torsion elements $x_{1}, \ldots, x_{k} \in Q,\left|x_{1} \cdots x_{k}\right|$ is a divisor of $\operatorname{lcm}\left\{\left|x_{1}\right|, \ldots,\left|x_{k}\right|\right\}$, no matter how $x_{1} \cdots x_{k}$ is parenthesized.

Proof. We have $(x y)^{n}=x^{n} y^{n}$ immediately from commutativity and diassociativity, and so (i) follows. If $x_{1}, \ldots, x_{k}$ are torsion elements, let $n=\operatorname{lcm}\left\{\left|x_{1}\right|, \ldots,\left|x_{k}\right|\right\}$. Then $\left(x_{1} \cdots x_{k}\right)^{n}=x_{1}^{n} \cdots x_{k}^{n}$, where the two products are parenthesized in analogous way. Since $x_{j}^{n}=1$ for each $j$, we have (ii).

For each $x$ in a loop $Q$, the left translation $L_{x}$ and the right translation $R_{x}$ are permutations of $Q$ defined, respectively, by $L_{x} y:=x y$ and $R_{x} y:=y x$ for all $y \in Q$. The inner mapping group $\operatorname{Inn}(Q)$ of a loop $Q$ is the stabilizer of the neutral element 1 in the group generated by all left and right translations. $\operatorname{Inn}(Q)$ is generated by all permutations of the forms $R_{x}^{-1} L_{x}, L_{x y}^{-1} L_{x} L_{y}$ and $R_{y x}^{-1} R_{x} R_{y}[2]$.

Recall that a subloop $P \leq Q$ is normal in $Q$ if and only if $\varphi P \subseteq P$ for all $\varphi \in \operatorname{Inn}(Q)$, that is, if and only if $P$ is invariant under the action of $\operatorname{Inn}(Q)$. With this characterization of normality, the following is obvious.

Lemma 2.2. Let $\left\{P_{i}\right\}_{i=1}^{\infty}$ be a sequence of normal subloops of a loop $Q$ satisfying $P_{i} \leq P_{i+1}$ for each $i$. Then $\bigcup_{i=1}^{\infty} P_{i}$ is a normal subloop.

Lemma 2.3. Let $Q$ be a commutative diassociative loop. Then for each prime $p, Q_{(p)} \unlhd Q$.

Proof. By Lemma 2.1, $Q_{\left[p^{m}\right]} \unlhd Q$ for each $m \geq 0$. Since $Q_{\left[p^{m}\right]} \leq Q_{\left[p^{m+1}\right]}$ for each $m$, and also $Q_{(p)}=\bigcup_{m \geq 0} Q_{\left[p^{m}\right]}$, we have $Q_{(p)} \unlhd Q$ by Lemma 2.2

Lemma 2.4. Let $Q$ be a commutative diassociative loop. If $m, n$ are relatively prime positive integers, then $Q_{[m n]}=Q_{[m]} Q_{[n]}$, a direct product.

Proof. If $x \in Q_{[m]}$ and $y \in Q_{[n]}$, then $(x y)^{m n}=x^{m n} y^{m n}=1$, and so $Q_{[m]} Q_{[n]} \subseteq Q_{[m n]}$. Now fix $z \in Q_{[m n]}$ and choose $r, s$ so that $m r+n s=1$. Then $z=z^{n s} z^{m r}$. Since $z^{n s} \in Q_{[m]}$ and $z^{m r} \in Q_{[n]}$, we have the other inclusion. The product is direct because each $Q_{[j]}$ is normal (Lemma 2.1) and $Q_{[m]} \cap Q_{[n]}=\{1\}$.

Theorem 2.5 (Bruck and Paige [3]). A torsion, commutative diassociative loop is the direct product of its p-primary components, that is, a direct product of commutative diassociative p-loops. 
MICHAEL K. KINYON AND PETR VOJTĚCHOVSKÝ

Proof. Let $Q$ be a torsion, commutative diassociative loop. In view of Lemma 2.3 , it remains to show that $Q=\left\langle Q_{(p)}\right| p$ prime $\rangle$, and $Q_{(p)} \cap\left\langle Q_{(q)}\right| q \neq p, q$ a prime $\rangle=1$.

Fix $x \in Q$ with $x \neq 1$. Since $Q$ is torsion, $x \in Q_{[n]}$ for some $n>0$. By Lemma 2.4 and induction, $Q_{[n]}=Q_{\left[p_{1}^{\left.a_{1}\right]}\right.} \cdots Q_{\left[p_{k}^{\left.a_{k}\right]}\right.}$ (direct product) where $n=p_{1}^{a_{1}} \cdots p_{k}^{a_{k}}$ for some distinct primes $p_{i}$ and exponents $a_{i}>0$. Since $Q_{\left[p_{i}^{a_{i}}\right]} \subseteq Q_{\left(p_{i}\right)}$, we have $x \in Q_{\left(p_{1}\right)} \cdots Q_{\left(p_{k}\right)}$. This shows $Q=\left\langle Q_{(p)}\right| p$ prime $\rangle$.

Now assume that $x \in Q_{(p)} \cap\left\langle Q_{(q)} \mid q \neq p\right\rangle$. Then $x \in\left\langle Q_{\left(q_{1}\right)}, \ldots, Q_{\left(q_{k}\right)}\right\rangle$ for some $q_{i} \neq p$. Since all $Q_{\left(q_{i}\right)}$ are normal in $Q$ by Lemma 2.1, we have $\left\langle Q_{\left(q_{1}\right)}, \ldots, Q_{\left(q_{k}\right)}\right\rangle=Q_{\left(q_{1}\right)} \cdots Q_{\left(q_{k}\right)}$. Thus $x=x_{1} \cdots x_{k}$, where $x_{i} \in Q_{\left(q_{i}\right)},\left|x_{i}\right|=q_{i}^{a_{i}}$, and the product $x_{1} \cdots x_{k}$ is parenthesized in some way. By Lemma 2.1] $|x|$ is a divisor of $q_{1}^{a_{1}} \cdots q_{k}^{a_{k}}$. But $|x|$ is also a power of $p$, so we conclude that $x=1$.

The nucleus and center of a loop $Q$ are the sets

$$
\begin{aligned}
& N(Q)=\{a \in Q \mid a \cdot x y=a x \cdot y, x \cdot a y=x a \cdot y, x \cdot y a=x y \cdot a, \forall x, y \in Q\}, \\
& Z(Q)=N(Q) \cap\{a \in Q \mid a x=x a, \forall x \in Q\} .
\end{aligned}
$$

The nucleus is a subloop of $Q$, but is not necessarily normal. The center is a normal subloop of any loop. In a commutative loop, the center and nucleus coincide.

Note that if $Q=\prod_{i} Q_{i}$ then $Z(Q)=\prod_{i} Z\left(Q_{i}\right)$. It is now easy to see what happens if we impose the condition that $x^{n}$ is central in torsion commutative diassociative loops.

Theorem 2.6. Let $n>0$ be a fixed integer, and let $p_{1}^{a_{1}} \cdots p_{k}^{a_{k}}$ be a prime factorization of $n$. Let $Q$ be a torsion commutative diassociative loop with each $x^{n} \in Z(Q)$. Then $Q$ is a direct product of commutative diassociative $p_{i}$-loops in which $p_{i}^{a_{i}}$ th powers are central with an abelian group in which each element has order prime to $n$.

Proof. Let $Q$ be a torsion commutative diassociative loop. Let $x \in Q_{\left(p_{i}\right)}$ and $m=n / p_{i}^{a_{i}}$. Since $|x|=p_{i}^{b_{i}}$ for some $b_{i}, m$ and $|x|$ are relatively prime, and so $x^{m}$ is a generator of $\langle x\rangle$. In particular, $x=x^{r m}$ for some $r$. Thus $x^{p_{i}^{a_{i}}}=x^{r n} \in Z(Q) \cap Q_{\left(p_{i}\right)}=Z\left(Q_{\left(p_{i}\right)}\right)$, where the last equality holds because $Q$ is a direct product of its $p$-primary components (Theorem 2.5).

Conversely, let $Q$ be a direct product of an abelian group $G$ and diassociative $p_{i}$-loops $Q_{i}$ in which $p_{i}^{a_{i}}$ th powers are central. Then clearly $x^{n} \in Z(G) \cap \bigcap_{i} Z\left(Q_{i}\right)=Z(Q)$.

\section{Moufang Elements}

There are various instances of diassociativity to which we will need to make specific reference. The inverse property (IP) is defined by any two of the following equations (which together imply the third):

(LIP) $\quad x^{-1} \cdot x y=y$,

(RIP) $\quad x y \cdot y^{-1}=x$,

(AAIP) $\quad(x y)^{-1}=y^{-1} x^{-1}$.

These are known, respectively, as the left inverse, right inverse, and antiautomorphic inverse properties.

Remark 3.1. Not all loops have two-sided inverses. Given a loop $Q$ and $x \in Q$, there are unique $x^{\lambda}, x^{\rho} \in Q$ such that $x^{\lambda} x=x x^{\rho}=1$. Then one can say that $Q$ has the inverse property if $x^{\lambda} \cdot x y=y x \cdot x^{\rho}=y$ for all $x, y \in Q$. But these identities imply $x^{\lambda}=x^{\rho}=x^{-1}$, so the inverse property can equivalently be stated as above. Moreover, in the commutative case, which we deal with in 4 we get $x^{\lambda}=x^{\rho}=x^{-1}$ for free.

We will also need the left alternative, right alternative, and flexible laws:

(LALT) $\quad x \cdot x y=x^{2} y$,

(RALT)

$x y \cdot y=x y^{2}$,

(FLEX)

$x \cdot y x=x y \cdot x$.

Loops satisfying both (LALT) and (RALT) are called alternative. 
Moufang loops are RIF loops, but flexible C-loops are not necessarily RIF. Both are included in a larger variety called $A R I F$ loops ("Almost RIF"), which are defined to be flexible loops satisfying either, and hence both, of the identities

$$
(\mathrm{ARIF} 1) \quad x(y x y \cdot z)=x y x \cdot y z \quad(\mathrm{ARIF} 2) \quad(z \cdot y x y) x=z y \cdot x y x
$$

These loops were introduced in [8], and the main result of that paper was the following.

Proposition 3.2. Every ARIF loop, and hence every RIF loop, is diassociative.

We will use Proposition 3.2 freely throughout what follows.

Recall that an autotopism of a loop $Q$ is a triple $(f, g, h)$ of permutations of $Q$ satisfying $f(x) g(y)=h(x y)$ for all $x, y \in Q$. Observe:

Proposition 3.3. Let $Q$ be an IP loop, let $J: Q \rightarrow Q ; x \mapsto x^{-1}$ denote the inversion mapping, and let $f, g, h$ be permutations of $Q$. The following are equivalent:

(i) $(f, g, h)$ is an autotopism,

(ii) $(J f J, h, g)$ is an autotopism,

(iii) $(h, J g J, f)$ is an autotopism.

We assume for the rest of this section that the flexible law holds. (We make this assumption to keep the situation manageable, although many of our arguments would work without it, too.)

There are thus 3 distinct Moufang identities (MFG), each with three variables. We now consider elements defined by fixing a variable in a Moufang identity. In anticipation of Lemma 3.5 below, we group the various possibilities as follows:

$$
\begin{aligned}
& \left(M_{0}\right) \quad c \cdot x y \cdot c=c x \cdot y c, \quad c(x \cdot c y)=c x c \cdot y, \quad(y c \cdot x) c=y \cdot c x c, \\
& \left(M_{1}\right) \\
& \left(M_{2}\right) \\
& \left(M_{3}\right) \\
& x c \cdot y x=x \cdot c y \cdot x, \quad x y x \cdot c=x(y \cdot x c), \\
& x y \cdot c x=x \cdot y c \cdot x, \quad c \cdot x y=(c x \cdot y) x .
\end{aligned}
$$

Each of these equations is assumed to be universally quantified in the variables $x$ and $y$.

We can view these identities as nine possibly different definitions of "Moufang elements." A natural question then is:

Problem 3.4. What are all the implications among the nine definitions of Moufang elements in the variety of flexible loops?

Without additional assumptions, we are not able to establish a single implication. However, in the IP case we have:

Lemma 3.5. For an element $c$ of a flexible IP loop $Q$,

(i) the equations $\left(M_{0}\right)$ are equivalent,

(ii) the equations $\left(M_{1}\right)$ are equivalent,

(iii) the equations $\left(M_{2}\right)$ are equivalent,

(iv) the equations $\left(M_{3}\right)$ are equivalent.

Proof. For (i): In IP loops, we have $J L_{x} J=R_{x}^{-1}$. Now the three equations are equivalent, respectively, to $\left(L_{c}, R_{c}, L_{c} R_{c}\right)$ being an autotopism, to $\left(R_{c} L_{c}, L_{c}^{-1}, L_{c}\right)$ being an autotopism, and to $\left(R_{c}^{-1}, L_{c} R_{c}, R_{c}\right)$ being an autotopism. The desired equivalence then follows from Proposition 3.3 applied to $f=L_{c}, g=R_{c}$ and $h=L_{c} R_{c}$.

For (ii): If $x c x \cdot y=x(c \cdot x y)$ holds, then replace $y$ with $x^{-1}\left(c^{-1} \cdot x^{-1} y^{-1}\right)=[(y x \cdot c) x]^{-1}$ to get $x c x \cdot[(y x \cdot c) x]^{-1}=x\left(c \cdot x\left[x^{-1}\left(c^{-1} \cdot x^{-1} y^{-1}\right)\right]\right)=y^{-1}$. Thus $x c x=y^{-1} \cdot(y x \cdot c) x$, and so $(y x \cdot c) x=y \cdot x c x$. The reverse implication follows from the mirror of this argument.

For (iii): if $x c \cdot y x=x \cdot c y \cdot x$ holds, then $x c \cdot\left(c^{-1} \cdot x^{-1} y^{-1} x^{-1}\right) x=x \cdot x^{-1} y^{-1} x^{-1} \cdot x=$ $y^{-1}$, using the IP. Thus $c^{-1} \cdot x^{-1} y^{-1} x^{-1}=\left(c^{-1} x^{-1} \cdot y^{-1}\right) x^{-1}$, and then using (AAIP) gives $x y x \cdot c=x(y \cdot x c)$. Conversely, if $x y x \cdot c=x(y \cdot x c)$, then following the argument in reverse 
gives $x c \cdot\left(c^{-1} \cdot x^{-1} y^{-1} x^{-1}\right) x=y^{-1}$. Replacing $y$ with $x^{-1} \cdot y^{-1} c^{-1} \cdot x^{-1}$ and using (AAIP) gives $x c \cdot y x=x \cdot c y \cdot x$.

Finally, the mirror of the proof of (iii) proves (iv).

For a flexible IP loop $Q$, let $M_{i}(Q), i=0, \ldots, 3$ denote the sets of elements satisfying, respectively, $\left(M_{i}\right), i=0, \ldots, 3$. When the underlying loop $Q$ is clear, as will usually be the case, we abbreviate $M_{i}=M_{i}(Q)$.

Elements of $M_{0}$ are known as Moufang elements ([2], p. 113). This definition is motivated by isotopy considerations; an element of an IP loop is contained in $M_{0}$ if and only if the loop isotope defined by that element has the IP. See [2] for details.

Lemma 3.6. Let $Q$ be a flexible, IP loop. Then

(i) $M_{0}$ is a subloop,

(ii) $c \in M_{1}$ if and only if $c^{-1} \in M_{1}$,

(iii) $c \in M_{2}$ if and only if $c^{-1} \in M_{3}$.

Proof. Part (i) is ([2], Chap. VII, Lemma 2.2). The rest follows immediately from (AAIP).

In a flexible IP loop which is not left alternative, the neutral element 1 satisfies $1 \in M_{0}$, but $1 \notin M_{1}$. The smallest order for which such a loop exists is 7 (this fact can be checked by computer with the help of any library of small loops, for instance the one found in the GAP [5] package LOOPS [11, or with a model builder, such as [9]): For instance, $5 \cdot 1(5 \cdot 6)=5 \cdot 3=7$, but

\begin{tabular}{c|ccccccc}
$\cdot$ & 1 & 2 & 3 & 4 & 5 & 6 & 7 \\
\hline 1 & 1 & 2 & 3 & 4 & 5 & 6 & 7 \\
2 & 2 & 3 & 1 & 6 & 7 & 5 & 4 \\
3 & 3 & 1 & 2 & 7 & 6 & 4 & 5 \\
4 & 4 & 7 & 6 & 5 & 1 & 2 & 3 \\
5 & 5 & 6 & 7 & 1 & 4 & 3 & 2 \\
6 & 6 & 4 & 5 & 3 & 2 & 7 & 1 \\
7 & 7 & 5 & 4 & 2 & 3 & 1 & 6
\end{tabular}

$(5 \cdot 1 \cdot 5) \cdot 6=4 \cdot 6=2$.

Lemma 3.7. In a flexible, alternative, IP loop, $M_{0} \subseteq M_{1}$.

Proof. If $c \in M_{0}$, then $c^{-1} \in M_{0}$ (since $M_{0}$ is a subloop), and so for all $x, y$,

$$
\begin{aligned}
x \cdot y c^{-1} y & \stackrel{\text { LIP }}{=} x \cdot\left[\left(c \cdot c^{-1} y\right) \cdot c^{-1} y\right] \stackrel{\text { RALT, LIP }}{=} c^{-1} \cdot c\left[x \cdot c\left(c^{-1} y\right)^{2}\right] \\
& \stackrel{c \in M_{0}}{=} c^{-1}\left[c x c \cdot\left(c^{-1} y\right)^{2}\right] \stackrel{\text { RALT }}{=} c^{-1}\left[\left(c x c \cdot c^{-1} y\right) \cdot c^{-1} y\right] \\
& \stackrel{c \in M_{0}}{=} c^{-1}\left[\left(c \cdot x\left(c \cdot c^{-1} y\right)\right) \cdot c^{-1} y\right] \stackrel{\text { LIP }}{=} c^{-1}\left[(c \cdot x y) \cdot c^{-1} y\right] \\
& c^{-1} \stackrel{\in}{=} M_{0} c^{-1}(c \cdot x y) c^{-1} \cdot y \stackrel{\text { LIP }}{=}\left(x y \cdot c^{-1}\right) y .
\end{aligned}
$$

Thus $c^{-1} \in M_{1}$, and so $c \in M_{1}$ (Lemma [3.6).

Problem 3.8. Does there exist a diassociative loop in which $M_{0} \neq M_{1}$ ? A flexible, alternative, IP loop?

Lemma 3.9. In a flexible, IP loop, $M_{0} \cap M_{2}=M_{0} \cap M_{3}$.

Proof. Fix $c \in M_{0} \cap M_{2}$. We compute

$$
c[x \cdot y c \cdot x] c \stackrel{c \in M_{0}}{=} c x \cdot(y c \cdot x) c \stackrel{c \in M_{0}}{=} c x \cdot(y \cdot c x c) \stackrel{c \in M_{2}}{=}(c x \cdot y \cdot c x) c .
$$


Canceling $c$ on the right, and then multiplying on the left by $c^{-1}$ and using (LIP), we get

$$
x \cdot y c \cdot x=c^{-1}(c x \cdot y \cdot c x) \stackrel{c^{-1} \in M_{3}}{=}\left(c^{-1} \cdot c x\right) y \cdot c x \stackrel{\text { LIP }}{=} x y \cdot c x,
$$

where we have used Lemma 3.6 in the second step. Thus $c \in M_{3}$.

Conversely, if $c \in M_{0} \cap M_{3}$, then $c^{-1} \in M_{0} \cap M_{2}$ (Lemma 3.6), and so $c^{-1} \in M_{3}$ by the preceding paragraph. Thus $c \in M_{2}$ (Lemma 3.6 again). This completes the proof.

Problem 3.10. Does there exist a diassociative loop in which $M_{2} \neq M_{3}$ ? A flexible, alternative, IP loop? A flexible IP loop?

Theorem 3.11. In an $A R I F$ loop, $M_{2}=M_{3} \subseteq M_{0}=M_{1}$.

Proof. If $c \in M_{1}$, then

$$
c \cdot x(c \cdot y) \stackrel{\text { LIP }}{=} c \cdot x\left(c \cdot\left[x \cdot x^{-1} y\right]\right) \stackrel{c \in M_{1}}{=} c\left(x c x \cdot x^{-1} y\right) \stackrel{\text { ARIF1 }}{=} c x c \cdot\left[x \cdot x^{-1} y\right] \stackrel{\text { LIP }}{=} c x c \cdot y .
$$

Therefore $c \in M_{0}$. We then have $M_{0}=M_{1}$ by Lemma 3.7 .

Now suppose $c \in M_{3}$. Then

$$
\begin{aligned}
y^{-1} x y^{-1} \cdot y\left(y^{-1} x \cdot c\right) y & \stackrel{c \in M_{3}}{=} y^{-1} x y^{-1} \cdot\left[\left(y \cdot y^{-1} x\right) \cdot c y\right] \stackrel{\text { LIP }}{=} y^{-1} x y^{-1} \cdot[x \cdot c y] \\
& \stackrel{\text { ARIF1 }}{=} y^{-1}\left[x y^{-1} x \cdot c y\right] \stackrel{\text { LIP, RALT }}{=} y^{-1}\left[\left(y \cdot\left(y^{-1} x\right)^{2}\right) \cdot c y\right] \\
& \stackrel{c \in M_{3}}{=} y^{-1}\left[y \cdot\left(y^{-1} x\right)^{2} c \cdot y\right] \stackrel{\text { LIP }}{=}\left(y^{-1} x\right)^{2} c \cdot y .
\end{aligned}
$$

Replacing $x$ with $y x$ and using (LIP), we have $x y^{-1} \cdot[y \cdot x c \cdot y]=x^{2} c \cdot y$, and so by (RALT),

$$
\begin{aligned}
(x \cdot x c) y \cdot x c & =\left(x y^{-1} \cdot[y \cdot x c \cdot y]\right) \cdot x c \stackrel{\text { ARIF } 2}{=} x(x c \cdot y \cdot x c) \\
& \stackrel{\operatorname{RIP}}{=}\left(x c \cdot c^{-1}\right)[x c \cdot y \cdot x c]^{c^{-1} \in M_{2}} x c \cdot c^{-1}(x c \cdot y) \cdot x c
\end{aligned}
$$

where we use Lemma 3.6 in the last step. Canceling $x c$ on the right and replacing $x$ with $x c^{-1}$ and using (RIP), we get $x c^{-1} x \cdot y=x\left(c^{-1} \cdot x y\right)$. Thus $c^{-1} \in M_{1}$, and so $c \in M_{1}$ by Lemma 3.6. This establishes $M_{3} \subseteq M_{1}=M_{0}$. By Lemma 3.6. we thus also have $M_{2} \subseteq M_{1}=M_{0}$. By Lemma 3.9. $M_{2}=M_{3}$. This completes the proof.

Problem 3.12. Is there an ARIF loop in which $M_{2} \neq M_{1}$ ?

Problem 3.12 has a negative answer for the two major subvarieties of the ARIF variety, namely RIF loops (Theorem 3.13) and flexible C-loops (Corollary 3.17).

Theorem 3.13. In a RIF loop, $M_{i}=M_{j}$ for all $i, j \in\{0,1,2,3\}$.

Proof. In view of Theorem 3.11, it is enough to show that $M_{0} \subseteq M_{2}$.

In the RIF identity (RIF1), replace $x$ with $x y^{-1}$, use (RIP), and then replace $y$ with $y^{-1}$ to get $x z x=x y \cdot\left[y^{-1}(z \cdot x y) y^{-1}\right]$. Now assume $c \in M_{0}$, and set $z=c u$ and $y=c$ to obtain

$$
x \cdot c u \cdot x=x c \cdot\left(c^{-1}(c u \cdot x c) c^{-1}\right) \stackrel{c \in M_{0}}{=} x c \cdot\left(c^{-1}(c \cdot u x \cdot c) c^{-1}\right) \stackrel{\text { LIP, RIP }}{=} x c \cdot u x .
$$

Thus $c \in M_{2}$.

An element $c$ of a loop $Q$ is a $C$ element if it satisfies

$$
x(c \cdot c y)=(x c \cdot c) y
$$

for all $x, y \in Q$. Note that $\mathrm{C}$ elements satisfy $c \cdot c x=c^{2} x$ and $x c \cdot c=x c^{2}$ for all $x \in Q$, which we use below without reference.

Let $C_{0}=C_{0}(Q)$ denote the set of all $\mathrm{C}$ elements of $Q$. Chein [4] showed the following:

Proposition 3.14. In an IP loop $Q, c \in C_{0}(Q)$ if and only if $c^{2} \in N(Q)$.

Lemma 3.15. In a flexible IP loop, $C_{0} \cap M_{0} \subseteq C_{0} \cap M_{2}=C_{0} \cap M_{3}$. 
Proof. If $c \in C_{0} \cap M_{2}$, then $c^{-1} \in M_{3}$ (Lemma 3.6), and so

$$
(c x \cdot y) x \stackrel{\text { LIP }}{=}\left(c^{2} \cdot c^{-1} x\right) y \cdot x \stackrel{c^{2} \in N}{=} c^{2} \cdot\left(c^{-1} x \cdot y\right) x \stackrel{c^{-1} \in M_{3}}{=} c^{2} \cdot\left(c^{-1} \cdot x y x\right) \stackrel{\text { LIP }}{=} c \cdot x y x .
$$

Thus $c \in M_{3}$. Therefore $C_{0} \cap M_{2} \subseteq C_{0} \cap M_{3}$ and Lemma 3.6 gives the reverse inclusion.

Now suppose $c \in C_{0} \cap M_{0}$. Then

$$
c x \cdot y c \cdot c x \stackrel{c \in M_{0}}{=}(c \cdot x y \cdot c) \cdot c x \stackrel{c \in M_{0}}{=} c\left(x y \cdot c^{2} x\right) \stackrel{c^{2} \in N}{=} c\left(x \cdot y c^{2} \cdot x\right) .
$$

Replace $y$ with $y c^{-1}$, use (RIP), and multiply on the right by $c$ :

$$
(c x \cdot y \cdot c x) c=c(x \cdot y c \cdot x) c \stackrel{c \in M_{0}}{=} c x \cdot(y c \cdot x) c \stackrel{c \in M_{0}}{=} c x \cdot y(c x \cdot c) .
$$

Replace $x$ with $c^{-1} x$ and use (LIP) to get $x y x \cdot c=x(y \cdot x c)$, that is, $c \in M_{2}$.

Theorem 3.16. In an ARIF loop, $C_{0} \cap M_{i}=C_{0} \cap M_{j}$ for all $i, j \in\{0,1,2,3\}$.

Proof. This follows immediately from Theorem 3.11 and Lemma 3.15

Corollary 3.17. In a flexible C-loop, $M_{i}=M_{j}$ for all $i, j \in\{0,1,2,3\}$.

\section{Commutative RIF loops}

We begin with some characterizations of the variety of commutative RIF loops.

Lemma 4.1. A loop $Q$ is a commutative RIF loop if and only if it is an IP loop satisfying the identity

$$
x\left(y^{2} \cdot x z\right)=(x y)^{2} z
$$

for all $x, y, z \in Q$.

Proof. In a commutative, alternative loop, we have

$$
(x y \cdot z) \cdot x y=x y \cdot(x y \cdot z)=(x y)^{2} z \quad \text { and } \quad x \cdot y(z x \cdot y)=x \cdot y(y \cdot x z)=x\left(y^{2} \cdot x z\right) .
$$

In RIF loops, the left hand sides are equal, and since such loops are diassociative, it follows that commutative RIF loops satisfy (CRIF). To complete the proof, it is enough to show that an IP loop satisfying (CRIF) is alternative and commutative. Taking $y=1$ in (CRIF), we get $x \cdot x z=x^{2} z$ which is (LALT). By (AAIP), any identity in an IP loop is equivalent to its mirror, so we also have $z x \cdot x=z x^{2}$, that is, (RAlt). Taking $z=1$ in (CRIF) gives $x \cdot y^{2} x=(x y)^{2}$, which is equivalent to $y^{2} x=x^{-1}(x y)^{2}$. Applying (LALT) and (RALt), we have $y \cdot y x=\left(x^{-1} \cdot x y\right) \cdot x y=y \cdot x y$. Canceling, it follows that $Q$ is commutative.

The identity (CRIF) has appeared in the literature before in other contexts. It plays a role in the theory of, for instance, Bruck loops [7].

The following is evidence of the naturality of the variety of commutative RIF loops. Among other things, it shows that passing from RIF to ARIF adds no generality in the commutative case.

Theorem 4.2. For a commutative loop $Q$, the following are equivalent.

(i) $Q$ is a RIF loop,

(ii) $Q$ is an ARIF loop.

(iii) $Q$ is an alternative, IP loop with each $x^{2} \in M_{0}(Q)$,

(iv) $Q$ satisfies (CRIF), 
Proof. (i) $\Longrightarrow$ (ii) holds even in the noncommutative case 8 .

For (ii) $\Longrightarrow$ (iii): By Proposition $3.2, Q$ is diassociative, and we freely use this and commutativity in the following calculation:

$$
\begin{aligned}
\left(z^{2} \cdot x^{2} y\right) x^{-1} & =L_{z^{2} \cdot x^{2} y} L_{x^{2} y}\left(y^{-1} x^{-3}\right) \stackrel{\mathrm{ARIF} 1}{=} L_{z} L_{z\left(x^{2} y\right)^{2}}\left(x^{3} y\right)^{-1}=L_{z} L_{x^{3} y}^{-1} L_{\left(x^{2} y\right)^{2}} z \\
& =L_{z} L_{x^{3} y}^{-1} L_{\left(x^{3} y\right)^{2} x^{-2}} L_{x^{-2}}\left(x^{2} z\right) \stackrel{\mathrm{ARIF} 1}{=} L_{z} L_{x^{3} y}^{-1} L_{x^{3} y} L_{x^{3} y \cdot x^{-4}}\left(x^{2} z\right) \\
& =L_{z} L_{x^{-1} y}\left(x^{2} z\right)=L_{z} L_{x^{2} z}\left(x^{-1} y\right) \stackrel{\mathrm{ARIF} 1}{=} L_{z^{2} x} L_{x}\left(x^{-1} y\right)=z^{2} x \cdot y .
\end{aligned}
$$

Thus $z^{2} \cdot x^{2} y=\left(z^{2} x \cdot y\right) x$, and so each $z^{2} \in M_{3}$. By Theorem 3.11, each $z^{2} \in M_{0}$.

For (iii) $\Longrightarrow$ (iv): If each $y^{2} \in M_{0}$, then by Lemma 3.7, each $y^{2} \in M_{1}$, and so $x\left(y^{2} \cdot x z\right)=$ $x y^{2} x \cdot z$ for all $x, y, z \in Q$. By commutativity and the alternative laws, $x y^{2} x=x(y \cdot x y)$. Now $x^{-1}(x y)^{2} \stackrel{\text { RALT }}{=}\left(x^{-1} \cdot x y\right) \cdot x y \stackrel{\text { LIP }}{=} y \cdot x y$, and so by $(\operatorname{LIP}),(x y)^{2}=x(y \cdot x y)$. Thus $x\left(y^{2} \cdot x z\right)=(x y)^{2} z$ for all $x, y, z \in Q$, that is, (CRIF) holds.

For (iv) $\Longrightarrow(\mathrm{i})$ : take $y=1$ in (CRIF) to get (LAlt), and by commutativity, (RAlt). Also,

$$
x y \cdot y \stackrel{\text { RALT }}{=} x y^{2}=x\left(y^{2} \cdot x x^{-1}\right) \stackrel{\text { CRIF }}{=}(x y)^{2} x^{-1} \stackrel{\text { LALT }}{=} x y \cdot\left(x y \cdot x^{-1}\right) .
$$

Canceling and using commutativity, we obtain $y=x^{-1} \cdot x y$, and so the IP holds. By Lemma 4.1. (i) holds.

The following is well-known, and holds in more generality than we give here.

Lemma 4.3. Let $Q$ be a commutative, IP loop. Then for every $x \in M_{0}(Q), x^{3} \in Z(Q)$.

Proof. By ([2, Chap. VII, Lemma 2.2), in an IP loop, for each $x \in M_{0}(Q)$, the inner mapping $R_{x}^{-1} L_{x}$ is a pseudoautomorphism with companion $x^{-3}$, that is, $x^{-3} \cdot(x \cdot y z) x^{-1}=\left[x^{-3} \cdot(x \cdot y) x^{-1}\right]$. $(x \cdot z) x^{-1}$, for all $y, z \in Q$. In the commutative case, this reduces to $x^{-3} \cdot y z=x^{-3} y \cdot z$, that is, $x^{-3} \in Z(Q)$.

Corollary 4.4. Let $Q$ be a commutative $R I F$ loop. Then $Q / Z(Q)$ has exponent 6.

Proof. By Theorem 4.2, every square is a Moufang element. Then by Lemma 4.3, every sixth power is central.

Recall that a Steiner loop is an IP loop of exponent 2, or equivalently, a C loop of exponent 2 [13. Such loops are commutative.

Theorem 4.5. Let $Q$ be a commutative RIF loop. Then:

(i) For each $x \in Q, x^{2} \in M_{0}(Q)$.

(ii) $M_{0}(Q)$ is a normal subloop of $Q$.

(iii) $Q / M_{0}(Q)$ is a $C$ loop of exponent 2, i.e., a Steiner loop.

Proof. Part (i) is Theorem 4.2(iii). For (ii): The set of Moufang elements is a subloop of any IP loop, so for (ii), only the normality requires a proof. Fix $b, c \in Q, a \in M_{0}(Q)$, and set $d=L_{b c}^{-1} L_{b} L_{c} a=(b c)^{-1}(b \cdot c a)$. We wish to show that $d \in M_{0}(Q)$. First, we compute

$$
b \cdot c a^{2}=b\left[a^{2} c^{2} \cdot c^{-1}\right]=b\left[(a c)^{2} \cdot b(b c)^{-1}\right]=(b \cdot a c)^{2}(b c)^{-1}=(b c)^{-1}(b c \cdot d)^{2}=d(b c \cdot d)=b c \cdot d^{2},
$$

where we have used (CRIF) in the third equality, and commutativity and diassociativity throughout. Thus $L_{b c}^{-1} L_{b} L_{c}\left(a^{2}\right)=d^{2}$. Now in RIF loops, inner mappings preserve inverses [8], and so $b \cdot c a^{-2}=b c \cdot d^{-2}$. Thus using $a^{-3} \in Z(Q)$ (Lemma 4.3), we have

$$
a^{-3} d=(b c)^{-1} \cdot a^{-3}(b \cdot c a)=(b c)^{-1}\left(b \cdot c a^{-2}\right)=(b c)^{-1}\left(b c \cdot d^{-2}\right)=d^{-2} .
$$

Therefore, $d^{3}=a^{3} \in Z(Q)$. On the other hand, $d^{2} \in M_{0}(Q)$, and since $Z(Q) \subseteq M_{0}(Q)$, we have $d=d^{3} d^{-2} \in M_{0}(Q)$. This completes the proof of normality.

Part (iii) then follows from (ii) and Theorem 4.2 
Next we turn to $\mathrm{C}$ elements. Although it is a bit of an aside to the rest of the development, we mention the following in passing.

Theorem 4.6. Let $Q$ be a commutative IP loop. Then $M_{0} \cap C_{0}=Z(Q)$.

Proof. If $a \in M_{0} \cap C_{0}$, then $a^{3} \in Z$ (Lemma 4.3) and $a^{2} \in Z$ (Proposition 3.14), and so $a=$ $a^{3} a^{-2} \in Z$. The other inclusion is clear.

For commutative RIF loops, the subset of $\mathrm{C}$ elements is well-structured.

Theorem 4.7. Let $Q$ be a commutative RIF loop. Then:

(i) For each $x \in Q, x^{3} \in C_{0}(Q)$.

(ii) $C_{0}(Q)$ is a normal subloop of $Q$.

(iii) $Q / C_{0}(Q)$ is a Moufang loop of exponent 3 .

Proof. Part (i) follows from Proposition 3.14 and Corollary 4.4

Now for $a, b \in C_{0}(Q),(a b)^{2}=a^{2} b^{2}$ by diassociativity, and so by Proposition 3.14, $a b \in C_{0}(Q)$. In addition, $a^{-1}$ is clearly in $C_{0}(Q)$, and so $C_{0}(Q)$ is a subloop. To show normality, fix $a \in C_{0}(Q)$, $b, c \in Q$, and set $d=L_{b c}^{-1} L_{b} L_{c} a=(b c)^{-1}(b \cdot c a)$. We wish to show $d \in C_{0}(Q)$. In RIF loops, inner mappings preserve inverses $\left[8\right.$, and so $b \cdot c a^{-1}=b c \cdot d^{-1}$. Using this and $a^{-2} \in Z(Q)$, we compute

$$
a^{-2} d=(b c)^{-1} \cdot a^{-2}(b \cdot c a)=(b c)^{-1}\left(b \cdot c a^{-1}\right)=(b c)^{-1} \cdot\left(b c \cdot d^{-1}\right)=d^{-1} .
$$

Thus $d^{2}=a^{2} \in Z(Q)$. Since $d^{3} \in C_{0}(Q)$ and $Z(Q) \subseteq C_{0}(Q)$, we have $d=d^{3} d^{-2} \in C_{0}(Q)$. This completes the proof of (ii).

Finally, $Q / C_{0}(Q)$ has exponent 3 by (i), and so by Theorem 4.5 , every element of $Q / C_{0}(Q)$ is Moufang. This proves (iii).

Finally, we have our decomposition theorem in the torsion case.

Theorem 4.8. Let $Q$ be a torsion, commutative RIF loop. Then $Q$ is the direct product of a $C$ 2-loop, a Moufang 3-loop, and an abelian group in which each element has order prime to 6 .

Proof. By Corollary 4.4, every sixth power is central. By Theorem 2.6. $Q$ is the direct product of a 2-loop, a 3-loop, and an abelian group in which each element has order prime to 6. Since every cube is a $\mathrm{C}$ element (Theorem 4.7), the 2-primary component is a $\mathrm{C}$ loop. Since every square is a Moufang element (Theorem 4.5), the 3-primary component is Moufang.

\section{Quasigroups associated to COMmutative RIF LOOPS}

Throughout this section, we will use multiplicative notation for quasigroups, and additive notation for loops. In particular, 0 is the neutral element, $-x$ is the inverse of $x$, and $x-y$ stands for $x+(-y)$ in loops.

A quasigroup $(Q, \cdot)$ is totally symmetric if it is commutative and satisfies the identity

$$
x \cdot x y=y
$$

for every $x, y \in Q$. An element $0 \in Q$ is an idempotent if $0^{2}=0$. Let $\mathbf{T} \mathbf{S}_{0}$ denote the category of totally symmetric quasigroups with a distinguished idempotent element (uniformly denoted by 0 ) preserved by morphisms.

A loop $(Q,+)$ with two-sided inverses has the weak inverse property if it satisfies the identity

$$
x-(y+x)=-y
$$

for every $x, y \in Q$. Let CWIP denote the category of commutative WIP loops.

Given a commutative quasigroup $(Q, \cdot)$ with an idempotent $0 \in Q$, define $\mathcal{L}(Q, \cdot)=(Q,+)$ by

$$
x+y=0 x \cdot 0 y .
$$

Conversely, given a loop $(Q,+)$ with neutral element 0 , define $\mathcal{Q}(Q,+)=(Q, \cdot)$ by

$$
x \cdot y=-x-y \text {. }
$$


It is then easy to show:

Proposition 5.1. $\mathcal{Q}$ is a functor $\boldsymbol{T} \boldsymbol{S}_{0} \rightarrow \boldsymbol{C W I P}$, and $\mathcal{L}$ is a functor $\boldsymbol{C W I P} \rightarrow \boldsymbol{T} \boldsymbol{S}_{0}$. Moreover, $\mathcal{L Q}$ is identical on $\boldsymbol{T} \boldsymbol{S}_{0}$, and $\mathcal{Q} \mathcal{L}$ is identical on $\boldsymbol{C W I P}$, so that the categories $\boldsymbol{T} \boldsymbol{S}_{0}, \boldsymbol{C W I P}$ are equivalent.

The equivalence of $\mathbf{T} \mathbf{S}_{0}$ and $\mathbf{C W I P}$ takes on a particularly nice form when restricted to certain subcategories.

In a quasigroup $Q$ let $\mathcal{I}(Q)$ denote the set of all idempotents of $Q$. In general, $\mathcal{I}(Q)$ need not be a subquasigroup of $Q$. A quasigroup is said to be idempotent if $Q=\mathcal{I}(Q)$.

A quasigroup $(Q, \cdot)$ is distributive if it satisfies

$$
x(y z)=x y \cdot x z, \quad(x y) z=x z \cdot y z
$$

for every $x, y, z \in Q$. Distributive quasigroups are idempotent. The following result is due to Bruck [1] (see also [12, Thm. V.2.16).

Proposition 5.2. Let $(Q,+)$ be a commutative Moufang loop of exponent 3 . Then $\mathcal{Q}(Q,+)$ is a totally symmetric, distributive quasigroup. Conversely, let $(Q, \cdot)$ be a totally symmetric, distributive quasigroup with a distinguished idempotent 0 . Then $\mathcal{L}(Q, \cdot)$ is a commutative Moufang loop of exponent 3 .

A quasigroup is said to be unipotent if $x^{2}=y^{2}$ for every $x, y$.

Proposition 5.3. Let $(Q,+)$ be a $C$ loop of exponent 2, i.e., a Steiner loop. Then $\mathcal{Q}(Q,+)$ is an unipotent, totally symmetric quasigroup. Conversely, let $(Q, \cdot)$ be a unipotent, totally symmetric quasigroup. Then $\mathcal{L}(Q, \cdot)$ is a Steiner loop.

Note that in a unipotent quasigroup there is a unique idempotent, namely $0=x^{2}=y^{2}$. In a unipotent, totally symmetric quasigroup, it is easy to see that the unique idempotent is a neutral element. Thus the equivalence of Proposition 5.3 is purely syntactical, since a unipotent, totally symmetric quasigroup is a Steiner loop. Put another way, the intersection of $\mathbf{T S}_{0}$ and $\mathbf{C W I P}$ is precisely the variety of Steiner loops with neutral 0, and each of the functors $\mathcal{Q}$ and $\mathcal{L}$ is identical on that intersection.

Our task is to generalize simultaneously Propositions 5.2 and 5.3 by finding the quasigroup counterpart of commutative RIF loops of exponent 6 under the functor $\mathcal{Q}$.

We introduce the following quasigroup axioms,

$$
\begin{gathered}
x^{2} x^{2}=x^{2}, \\
x\left(y^{2} \cdot x z\right)=(x y)^{2} z,
\end{gathered}
$$

noting that (Q2) is just another name for (CRIF), this time in quasigroups.

Lemma 5.4. A totally symmetric quasigroup satisfying (Q2) is distributive if and only if it is idempotent.

Proof. Only the sufficiency requires a proof. In the idempotent case, (Q2) is equivalent to $x(y$. $x z)=x y \cdot z$. Replacing $z$ with $x z$ and applying (TS), we obtain (D).

For $0 \in \mathcal{I}(Q)$, let $\mathcal{U}_{0}(Q)=\left\{x \in Q \mid x^{2}=0\right\}$.

Lemma 5.5. Let $Q$ be a totally symmetric quasigroup satisfying (Q1), (Q2). Then

(i) the squaring mapping $Q \rightarrow Q ; x \mapsto x^{2}$ is an endomorphism of $Q$ with image $\mathcal{I}(Q)$,

(ii) $\mathcal{I}(Q)$ is a distributive subquasigroup,

(iii) for each $0 \in \mathcal{I}(Q), \mathcal{U}_{0}(Q)$ is a unipotent subquasigroup, that is, a Steiner loop.

Proof. Set $z=x$ in (Q2) and cancel $x$ on both sides to obtain $x^{2} y^{2}=(x y)^{2}$. Thus squaring is an endomorphism. The image is a subset of $\mathcal{I}(Q)$ by $(\mathrm{Q} 1)$, and since every idempotent is trivially a square, the image coincides with $\mathcal{I}(Q)$. This establishes (i). Homomorphic images of quasigroups are subquasigroups, so (ii) follows from Lemma 5.4. Finally, (iii) follows from (i). 
Theorem 5.6. Let $(Q,+)$ be a commutative RIF loop of exponent 6 . Then $(Q, \cdot)=\mathcal{Q}(Q,+)$ is a totally symmetric quasigroup satisfying (Q1), (Q2).

Conversely, let $(Q, \cdot)$ be a totally symmetric quasigroup satisfying (Q1), (Q2), and let $0 \in Q$ be an idempotent. Then $(Q,+)=\mathcal{L}(Q, \cdot)$ is a commutative RIF loop of exponent 6 .

Proof. Let $(Q,+)$ be a commutative $\operatorname{RIF}$ loop of exponent 6 , and let $(Q, \cdot)=\mathcal{Q}(Q,+)$. Note that $x^{2}=-2 x$. Using diassociativity and the fact that $Q$ has exponent 6 , we compute

$$
x^{2} \cdot x^{2}=-(-2 x)-(-2 x)=4 x=-2 x=x^{2}
$$

for all $x, y \in Q$. Thus (Q1) holds. Next

$$
\begin{gathered}
x\left(y^{2} \cdot x z\right)=-x-(2 y-(-x-z))=-x+[-2 y+(-x-z)] \\
\stackrel{(\mathrm{CRIF})}{=} 2(-x-y)-z=(x y)^{2} z
\end{gathered}
$$

for all $x, y, z \in Q$, and so (Q2) holds.

Now let $(Q, \cdot)$ be a totally symmetric quasigroup satisfying (Q1), (Q2), and let $0 \in Q$ be an idempotent. (Idempotents exist by (Q1).) Let $(Q,+)=\mathcal{L}(Q, \cdot)$. First note that $2 x=(0 x)^{2}=0 x^{2}$ for all $x$ by Lemma 5.5(i). We use this in the following calculations. We verify (CRIF) as follows:

$$
\begin{aligned}
x+[2 y+(x+z)] & =0 x \cdot 0\left[\left(0 \cdot 0 y^{2}\right) \cdot 0(0 x \cdot 0 z)\right] \stackrel{(\mathrm{TS})}{=} 0 x \cdot 0\left[y^{2} \cdot 0(0 x \cdot 0 z)\right] \\
& \stackrel{(\mathrm{Q} 2)}{=} 0 x \cdot(0 y)^{2}(0 x \cdot 0 z) \stackrel{(\mathrm{Q} 2)}{=}(0 x \cdot 0 y)^{2} \cdot 0 z \stackrel{(\mathrm{TS})}{=}\left[0 \cdot 0(0 x \cdot 0 y)^{2}\right] \cdot 0 z \\
& =2(x+y)+z,
\end{aligned}
$$

where we used in the second step. By Theorem 4.2, $(Q,+)$ is a commutative RIF loop. We have

$$
6 x=2 x+(2 x+2 x)=\left(0 \cdot 0 x^{2}\right) \cdot 0\left[\left(0 \cdot 0 x^{2}\right) \cdot\left(0 \cdot 0 x^{2}\right)\right] \stackrel{(\mathrm{TS})}{=} x^{2} \cdot 0\left[x^{2} x^{2}\right] \stackrel{(\mathrm{Q} 1)}{=} x^{2} \cdot 0 x^{2} \stackrel{(\mathrm{TS})}{=} 0,
$$

and this completes the proof.

Theorem 5.7. Let $Q$ be a totally symmetric quasigroup satisfying (Q1), (Q2). Then for each $0 \in \mathcal{I}(Q), Q$ is a direct product of $\mathcal{I}(Q)$ and $\mathcal{U}_{0}(Q)$. Thus every totally symmetric quasigroup satisfying (Q1), (Q2) is a direct product of a distributive subquasigroup and a Steiner loop.

Proof. Let $(Q,+)=\mathcal{L}(Q, \cdot)$ be the associated commutative RIF loop of exponent 6 (Theorem 5.6). By Theorem 4.8, $(Q,+)$ is a direct product of a Moufang subloop $\left(Q_{1},+\right)$ of exponent 3 and a $\mathrm{C}$ subloop $\left(Q_{2},+\right)$ of exponent 2 , that is, a Steiner loop.

The subquasigroup $\left(Q_{1}, \cdot\right)=\mathcal{Q}\left(Q_{1},+\right)$ of $(Q, \cdot)$ is distributive (Proposition 5.2) and hence, idempotent. Thus $Q_{1} \subseteq \mathcal{I}(Q)$. On the other hand, if $c \in \mathcal{I}(Q)$, then

$$
c+(c+c)=0 c \cdot 0(0 c \cdot 0 c)=0 c \cdot 0\left(0^{2} c^{2}\right) \stackrel{(\mathrm{TS})}{=} 0 c \cdot c \stackrel{(\mathrm{TS})}{=} 0,
$$

and so $c \in Q_{1}$. Therefore $Q_{1}=\mathcal{I}(Q)$.

Next, the subquasigroup $\left(Q_{2}, \cdot\right)=\mathcal{Q}\left(Q_{2},+\right)$ is just $\left(Q_{2},+\right)$ itself in different notation. In particular, $Q_{2} \subseteq \mathcal{U}_{0}(Q)$. On the other hand, if $x \in \mathcal{U}_{0}(Q)$, then $x^{2}=0$, and so $x+x=0$, that is, $x \in Q_{2}$. Therefore $Q_{2}=\mathcal{U}_{0}(Q)$.

Finally, noting that the functor $\mathcal{Q}$ sends a direct product of commutative diassociative loops to a direct product of quasigroups, we have the desired result.

Remark 5.8. Steiner quasigroups are defined as idempotent, totally symmetric quasigroups. There is a one-to-one correspondence between Steiner quasigroups of order $n$ and Steiner loops of order $n+1$. (Given a Steiner quasigroup, introduce a new element 1, leave $x \cdot y$ intact for $x \neq y$, and set $x^{2}=1$. Conversely, given a Steiner loop with neutral element 1 , remove 1 , leave $x \cdot y$ intact for $x \neq y$, and set $x^{2}=x$.) Moreover, it is well-known that Steiner quasigroups are in one-to-one correspondence to Steiner triple systems. Are there interesting combinatorial structures associated to commutative RIF loops? 


\section{REFERENCES}

[1] R. H. Bruck, Some results in the theory of quasigroups, Trans. Amer. Math. Soc. 55 (1944), 19-52.

[2] R. H. Bruck, A Survey of Binary Systems, Springer-Verlag, 1971.

[3] R. H. Bruck and L. J. Paige, Loops whose inner mappings are automorphisms, Ann. of Math. (2) 63 (1956), 308-323.

[4] O. Chein, A short note on supernuclear (central) elements of inverse property loops. Arch. Math. (Basel) 33 (1979/80), 131-132.

[5] The GAP Group, GAP - Groups, Algorithms, and Programming, Version 4.4.9; 2006. http://www.gap-system.org

[6] J. Hart and K. Kunen, Single axioms for odd exponent groups, J. Automated Reasoning 14 (1995), 383-412.

[7] H. Kiechle, Theory of K-loops, Lecture Notes in Mathematics 1778, Springer, 2002.

[8] M. K. Kinyon, K. Kunen, and J. D. Phillips, A generalization of Moufang and Steiner loops, Algebra Universalis 48 (2002), 81-101.

[9] W. W. McCune, Mace4 Reference Manual and Guide, Tech. Memo ANL/MCS-TM-264, Mathematics and Computer Science Division, Argonne National Laboratory, Argonne, IL, August 2003. http://www.cs.unm.edu/ mccune/mace4/

[10] W. W. McCune, Prover9 Manual, http://www.cs.unm.edu/ mccune/prover9/

[11] G. P. Nagy and P. Vojtěchovský, LOOPS - a GAP package, version 1.4.0, Feb. 2007, http://www.math.du.edu/loops

[12] H. O. Pflugfelder, Quasigroups and Loops: Introduction, Sigma Series in Pure Math. 8, Heldermann Verlag, Berlin, 1990.

[13] J. D. Phillips and P. Vojtěchovský, C-loops: An introduction, Pub. Math. Debrecen 68 (2006), 115-137.

E-mail address: mkinyon@math.du.edu

URL: http://math.du.edu/ ${ }^{\sim m k i n y o n}$

E-mail address: petr@math.du.edu

URL: http://math.du.edu/ petr

Department of Mathematics, University of Denver, Denver, CO 80208 USA 